\title{
Complete genome sequence of bile-isolated Enterococcus avium strain 352
}

\author{
Tao Yu ${ }^{1,2}$, Lixiang Li $i^{1,2}$, Qilin Zhao ${ }^{3}$, Peng Wang ${ }^{1,2}$ and Xiuli Zuo ${ }^{1,2^{*}}$
}

\begin{abstract}
Background: Enterococcus avium is a Gram-positive pathogenic bacterium belonging to the family Enterobacteriaceae. E. avium can cause bacteremia, peritonitis, and intracranial suppurative infection. However, the mechanism of its pathogenesis and its adaptation to a special niche is still unclear.

Results: In this study, the E. avium strain 352 was isolated from human bile and whole genome sequencing was performed. The E. avium strain 352 consists of a circular 4,794,392 bp chromosome as well as an 87,705 bp plasmid. The GC content of the chromosome is 38.98\%. There are 4905 and 99 protein coding sequences in the chromosome and the plasmid, respectively. The genome of the E. avium strain 352 contains number of genes reported to be associated with bile adaption, including bsh, sbcC, mutS, nifl, galU, and hupB. There are also several virulence-associated genes including esp, fss 1, fss3, ecbA, bsh, lap, clpC, clpE, and clpP.
\end{abstract}

Conclusions: This study demonstrates the presence of various virulence factors of the E. avium strain 352, which has the potential to cause infections. Moreover, the genes involved in bile adaption might contribute to its ability to live in bile. Further comparative genomic studies would help to elucidate the evolution of pathogenesis of E. avium.

Keywords: E. avium, Virulence factors, Bile adaption

\section{Background}

Enterococcus avium is a Gram-positive bacterium of the genus Enterococcus and is most commonly found in birds. E. avium is also a cause of infectious diseases in humans including bacteremia, peritonitis, intracranial suppurative infection and osteomyelitis [1-5]. It was reported that $E$. avium is responsible for approximately $1 \%$ of infections in humans [3]. However, there is not much known about the mechanism of its pathogenesis.

Enterococcus avium was isolated from blood samples, fecal samples, spinal cords, jeotgals (a Korean fermented seafood), and scallop solutions [1, 3, 6, 7]. Thus, E. avium can adapt to various environments and this might be an important factor for its survival in humans and for subsequent infections. Currently, there are $8 \mathrm{draft}$ genomes of $E$. avium accessible on NCBI databases. However, no

\footnotetext{
*Correspondence: zuoxiuli@sdu.edu.cn

1 Department of Gastroenterology, Qilu Hospital, Shandong University,

107 Wenhuaxi Road, Jinan 250012, Shandong, People's Republic of China

Full list of author information is available at the end of the article
}

studies have analyzed these genomes for the niche adaptation of E. avium.

Here, we report the first whole genome sequence of $E$. avium. We also analyzed the virulence-associated genes and bile stress adaptation mechanism of the E. avium strain 352.

\section{Methods \\ Strain isolation and characterization}

The E. avium strain 352 was isolated from a bile sample of a cholelithiasis patient. This strain was cultivated on blood plate agar under anaerobic conditions at $37^{\circ} \mathrm{C}$ for $24 \mathrm{~h}$. This strain was identified by $16 \mathrm{~S}$ rRNA sequencing using the following primers including 27F (5'-AGA GTTTGATCCTGGCTCAG-3') and 1492R (5'-GGTTAC CTTGTTACGACTT-3'). The PCR products were subsequently sequenced, and these sequences were compared against the $16 \mathrm{~S}$ rRNA bacteria sequence database using BLAST from the NCBI website.

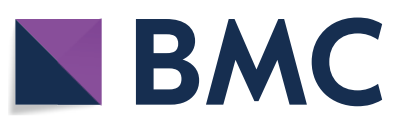

(c) The Author(s) 2019. This article is distributed under the terms of the Creative Commons Attribution 4.0 International License (http://creativecommons.org/licenses/by/4.0/), which permits unrestricted use, distribution, and reproduction in any medium, provided you give appropriate credit to the original author(s) and the source, provide a link to the Creative Commons license, and indicate if changes were made. The Creative Commons Public Domain Dedication waiver (http://creativecommons.org/ publicdomain/zero/1.0/) applies to the data made available in this article, unless otherwise stated. 


\section{Genome sequencing and de novo assembly}

The bacterial genomic DNA was extracted from overnight culture of the E. avium 352 using the Bacteria DNA Kit (OMEGA Bio-Tek Inc., Norcross, GA, USA) according to the manufacturer's instructions, and quality control was subsequently carried out using TBS-380 fluorometer (Turner BioSystems Inc., Sunnyvale, CA). Then, high qualified DNA sample (OD260/280 $=1.8$ $2.0,>6 \mu \mathrm{g})$ was utilized to construct a fragment library.

Genomic DNA (above $3 \mu \mathrm{g}$ ) was subjected to whole genome sequencing on an Illumina HiSeq Sequencer (PE150 mode) according to the sequencing protocol. Raw sequencing data was generated by Illumina base calling software CASAVA v1.8.2 (Illumina Inc. San Diego, CA, USA). Contamination reads, such as ones containing adaptors or primers were identified by Trimmomatic with default parameters. Clean data obtained by above quality control processes were used to do further analysis. Meanwhile, the whole-genome sequencing of E. avium 352 was also carried out on the single molecule real-time by the PacBio RS Platform (Pacific Biosciences of California, Inc., Menlo Park, CA, USA). A $20 \mathrm{~K}$ template library was generated and sequenced using standard methods.

The Illumina data were used to evaluate the complexity of the genome and correct the PacBio long reads. First, we used ABySS to peform genome assembly with multiple-Kmer parameters and obtained optimal results for the assembly [8]. Second, canu (https://github.com/ $\mathrm{marbl} / \mathrm{canu}$ ) was used to assemble the PacBio corrected long reads [9]. Finally, GapCloser software was subsequently applied to fill the remaining local inner gaps and correct the single base polymorphism for the final assembly results [10].

Gene annotation was determined by Annotation NCBI Prokaryotic Genome Annotation Pipeline [11]. Ribosomal RNA genes were detected by RNAmer 1.2 [12] and tRNA genes were recognized via tRNAscan SE v. 2.0 [13]. The circular genomic map was produced using CGView Server [14].

Phylogenetic analysis is based on orthologous genes. First, orthologous gene families were identified by the ORTHOMCL v2.0 program (reciprocal all-by-all BLASTP analysis) with an E-value of $10^{-5}$ [15]. Second, multiple alignments were generated with the MUSCLE v3.8.31 program, and the alignments were examined visually [16]. Third, the Maximum-likelihood (ML) methods were performed for the phylogenetic analyses using PhyML 3.0, and the model GTR + G was selected for ML analyses with 500 bootstrap replicates to calculate the bootstrap values [17]. The strains used for phylogenetic tree analysis included the E. avium strain ATCC 14025 (GCA000406965.1), the E. faecalis strain
ATCC 19433 (GCA000392875.1), the E. faecium strain DO (GCA000174395.2), the E. gilvus strain ATCC BAA350 (GCA_000394615.1), the E. pseudoavium strain CBA7133 (GCA 003386455.1), the E. sulfureus strain ATCC 49903 (GCA000407025.1), the E. raffinosus strain ATCC 49464 (GCA000393895.1), the E. gallinarum strain FDAARGOS_163 (GCA001558875.2), and the Vagococcus fluvialis strain DSM 5731 (GCA003337315.1). The putative virulence related genes were identified based on the whole genome of the E. avium strain 352 using the VFDB [18].

\section{Quality assurance}

A single colony of the E. avium strain 352 was repeatedly transferred to fresh brain heart infusion (BHI) medium to obtain pure cultures. Before DNA extraction, the identity of the strain was verified through $16 \mathrm{~S}$ rRNA gene sequencing. After the genome sequence was obtained, the $16 \mathrm{~S}$ rDNA gene was extracted from the genome using the RNAmmer 1.2 server and then confirmed through a BLAST search of the 16S rRNA gene against the NCBI microbial $16 \mathrm{~S}$ database.

\section{Results and discussion}

General genome features of the E. avium strain 352

Total of 46,188,978 raw reads were obtained by Illumina HiSeq Sequencer, and 45,357,196 high quality reads were generated after quality control processes. In addition, $168,754(1.26 \mathrm{~Gb})$ high-quality reads with an average read length of $7500 \mathrm{bp}$ and a 259 -fold coverage were generated by PacBio sequencer. These sequences were used to assemble the genome of the E. avium strain 352 and we obtained a circular chromosome without gap. The complete genome is $4.79 \mathrm{Mb}$ in size with a plasmid of $87.7 \mathrm{~kb}$ (Fig. 1) and the mean G + C content is $38.98 \%$. This genome contains 4905 predicted genes as well as 18 rRNA and 68 tRNA genes, while there were 99 predicted genes in the plasmid.

\section{Phylogenetic analysis}

The 16S rRNA gene sequence verified the taxonomic status of the E. avium strain 352 (data not shown). To further elucidate the phylogenetic relationships, whole genome DNA-sequence-based phylogenetic analysis was carried out (Fig. 2). The genome of a highly related and similar type of $E$. avium strain, E. avium strain ATCC 14025 , was selected as standard. The dendrogram of phylogenetic trees illustrated that the E. avium strain 352 was most closely related to the E. avium strain ATCC 14025. 


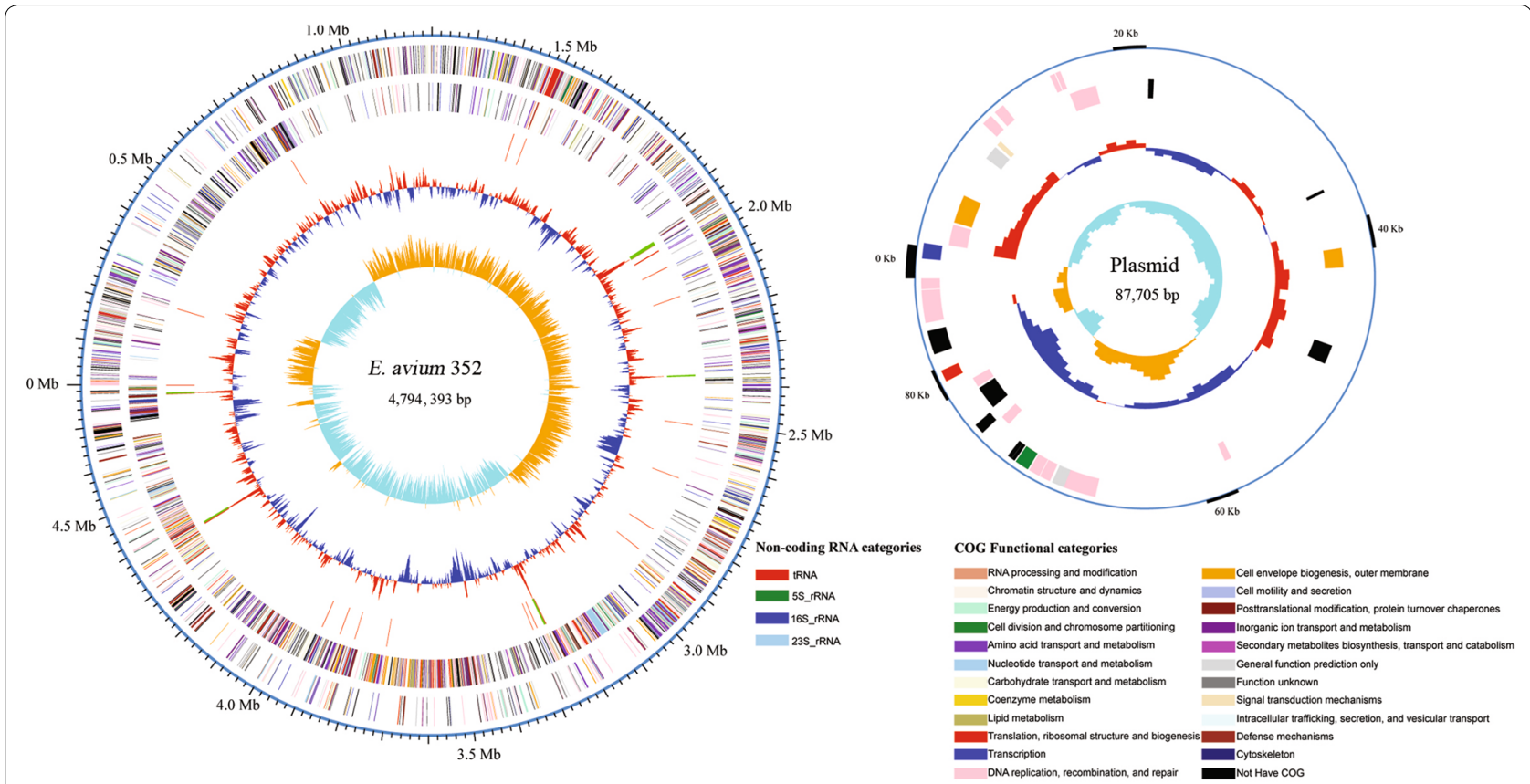

Fig. 1 Genome map of E. avium 352. Circles from the outside to inside showing: (1) DNA coordinates; (2, 3) function-based color-coded mapping of the CDSs predicted on the forward and reverse strands. Functions are color-coding; (4) tRNA genes and rRNA genes; (5) GC plot showing regions above the average (red) and below (blue); (6) GC skew

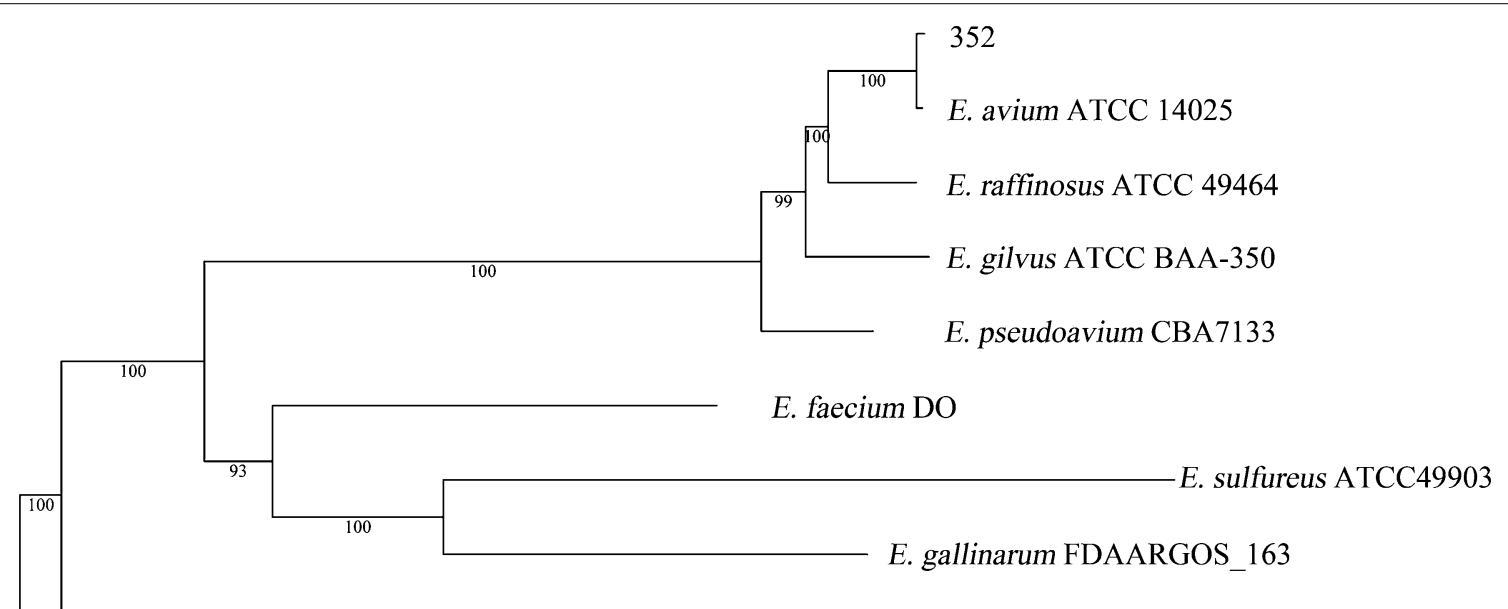

E. faecalis ATCC 19433

V. fluvialis DSM 5731

$0.01 \longmapsto$

Fig. 2 Phylogenetic analysis of E. avium 352

\section{Identification of genes related to bile stress}

Bile salts have potent antimicrobial activity via damaging membranes and DNA. Thus, bacteria must have intrinsic adapted mechanisms to survive in bile and subsequently cause biliary tract infections [19]. Genomic analysis of the E. avium strain 352 showed the presence of numerous genes that may determine its bile resistance properties (Table 1). The presence of the genes $s b c C$, mutS and nifI involved in bile resistance in Gram-positive bacteria was identified [19]. It is interesting that there were two 
Table 1 Putative genes for bile adaptation in E. avium 352

\begin{tabular}{ll}
\hline Gene name & Fuction/putative fuction \\
\hline hupB & HU family DNA-binding protein \\
galU & UDP-glucose-pyrophosphorylase \\
sbcC & Exonuclease SbcC \\
muts & DNA mismatch repair \\
bsh1 & Bile salt hydrolase \\
bsh2 & Bile salt hydrolase \\
nifl & Pyruvate: ferredoxin oxidoreductase
\end{tabular}

Table 2 Putative virulence associated genes in E. avium 352 predicted by VFDB

\begin{tabular}{llcl}
\hline $\begin{array}{l}\text { Gene } \\
\text { name }\end{array}$ & Function/putative function & Score & E value \\
\hline esp & Enterococcal surface protein & 3907 & 0 \\
fss3 & Enterococcus faecalis surface protein Fss3 & 3749 & 0 \\
ecbA & Collagen binding MSCRAMM & 3416 & 0 \\
bsh & Bile salt hydrolase & 274 & $6 \mathrm{e}-70$ \\
lap & Listeria adhesion protein & 129 & $2 \mathrm{e}-26$ \\
clpE & ATP-dependent protease & 127 & $9 \mathrm{e}-26$ \\
clpP & ATP-dependent c1p protease peoteolytic & 117 & $8 \mathrm{e}-23$ \\
& $\quad$ subunit & & \\
fss1 & Enterococcus faecalis surface protein Fss1 & 107 & $8 \mathrm{e}-20$ \\
clpC & Endopeptidase C1p ATP-binding chain C & 76 & $3 \mathrm{e}-10$ \\
\hline
\end{tabular}

bsh genes encoding bile salt hydrolase with a protein sequence identity of $92.9 \%$ in the genome of the E. avium strain 352. This result indicated that the BSH might be play an important role in niche-specific adaptation for bile [20]. There were also some genes, including galU and hupB, involved in bile resistance in Gram-negative bacteria [19]. Further studies are needed to verify its genetic properties and evolution traits.

\section{Analysis of virulence associated genes}

Further screening the genome of the E. avium strain 352 for putative virulence-associated genes was conducted by aligning gene sequences to the virulence factor database (Table 2). There are surface protein encoded genes including esp, fss 1 and $f s s 3$. The E. avium strain 352 also contains the conservative heat shock protein genes $c l p C$, $c l p E$, and $\operatorname{clpP}$ [21]. The $e c b A$ gene encoding a collagen binding MSCRAMM (acronym for microbial surface components recognizing adhesive matrix molecules) and gene lap encoding a listeria adhesion protein were found in the genome and might be contribute to adherence to the host tissue $[22,23]$. The $b s h$ gene encoding a bile salt hydrolase was also a virulence related factor in Listeria monocytogenes [24]. The clinical significance of this finding warrants further investigation.

\section{Authors' contributions}

TY and $L X L$ designed the study; $L X L$ and $Q L Z$ isolated and identified the $E$. avium 352; TY and LXL analyzed data and wrote the manuscript; PW revised the manuscript; XLZ support this study and revised the manuscript. All authors read and approved the final manuscript.

\section{Author details}

${ }^{1}$ Department of Gastroenterology, Qilu Hospital, Shandong University, 107 Wenhuaxi Road, Jinan 250012, Shandong, People's Republic of China. ${ }^{2}$ Laboratory of Translational Gastroenterology, Qilu Hospital, Shandong University, Jinan 250012, People's Republic of China. ${ }^{3}$ High School Attached To Shandong Normal University, Jinan 250012, People's Republic of China.

\section{Acknowledgements}

Not applicable.

\section{Competing of interests}

The authors declare that they have no competing interests.

\section{Availability of data and materials}

The completed genome sequence of E. avium 352 has been deposited into GenBank database with accession number CP034169 (chromosome) and

CP034168 (plasmid), respectively.

\section{Ethics approval and consent to participate}

This study was approved by the ethics committees in Qilu hospital of Shandong University.

\section{Funding}

This research was supported by the Key Research and Development Program of Shandong Province (2017CXGC1215) and National Natural Science Foundation of China (81600428).

\section{Publisher's Note}

Springer Nature remains neutral with regard to jurisdictional claims in published maps and institutional affiliations.

Received: 16 December 2018 Accepted: 23 March 2019

Published online: 24 April 2019

\section{References}

1. Cottagnoud P, Rossi M. Enterococcus avium osteomyelitis. Clin Microbiol Infect. 1998;4(5):290.

2. Na S, et al. Enterococcus avium bacteremia: a 12-year clinical experience with 53 patients. Eur J Clin Microbiol Infect Dis. 2012;31(3):303-10.

3. Okada A, Hangai M, Oda T. Bacteremia with an iliopsoas abscess and osteomyelitis of the femoral head caused by Enterococcus avium in a patient with end-stage kidney disease. Intern Med. 2015;54(6):669-74.

4. Yildirmak T, et al. Community-acquired intracranial suppurative infections: a 15-year report. Surg Neurol Int. 2014;5:142.

5. Ugur AR, et al. Enterococcus avium peritonitis in a child on continuous ambulatory peritoneal dialysis. Perit Dial Int. 2014;34(1):127-8.

6. Shin NR, et al. Isolation and characterization of human intestinal Enterococcus avium EFEL009 converting rutin to quercetin. Lett Appl Microbiol. 2016;62(1):68-74

7. Yang $\mathrm{H}$, et al. Accumulation of gamma-aminobutyric acid by Enterococcus avium 9184 in scallop solution in a two-stage fermentation strategy. Microb Biotechnol. 2016;9(4):478-85.

8. Jackman SD, et al. ABySS 2.0: resource-efficient assembly of large genomes using a Bloom filter. Genome Res. 2017;27(5):768-77.

9. Koren S, et al. Canu: scalable and accurate long-read assembly via adaptive k-mer weighting and repeat separation. Genome Res. 2017;27(5):722-36. 
10. Luo R, et al. SOAPdenovo2: an empirically improved memory-efficient short-read de novo assembler. Gigascience. 2012;1(1):18.

11. Tatusova T, et al. NCBI prokaryotic genome annotation pipeline. Nucleic Acids Res. 2016;44(14):6614-24.

12. Lagesen $\mathrm{K}$, et al. RNAmmer: consistent and rapid annotation of ribosomal RNA genes. Nucleic Acids Res. 2007;35(9):3100-8.

13. Lowe TM, Eddy SR. tRNAscan-SE: a program for improved detection of transfer RNA genes in genomic sequence. Nucleic Acids Res. 1997:25(5):955-64.

14. Grant JR, Stothard P. The CGView Server: a comparative genomics tool for circular genomes. Nucleic Acids Res. 2008;36(Web Server issue):W181-4.

15. Li L, Stoeckert CJ Jr, Roos DS. OrthoMCL: identification of ortholog groups for eukaryotic genomes. Genome Res. 2003;13(9):2178-89.

16. Edgar RC. MUSCLE: multiple sequence alignment with high accuracy and high throughput. Nucleic Acids Res. 2004;32(5):1792-7.

17. Guindon S, et al. New algorithms and methods to estimate maximumlikelihood phylogenies: assessing the performance of PhyML 3.0. Syst Biol. 2010;59(3):307-21.

18. Chen L, et al. VFDB 2016: hierarchical and refined dataset for big data analysis - 10 years on. Nucleic Acids Res. 2016;44(D1):D694-7.
19. Begley M, Gahan CG, Hill C. The interaction between bacteria and bile. FEMS Microbiol Rev. 2005;29(4):625-51.

20. Bi J, et al. Bile salt tolerance of Lactococcus lactis is enhanced by expression of bile salt hydrolase thereby producing less bile acid in the cells. Biotechnol Lett. 2016;38(4):659-65.

21. Cassenego AP, et al. The CtsR regulator controls the expression of $\mathrm{clpC}$, $\mathrm{clpE}$ and clpP and is required for the virulence of Enterococcus faecalis in an invertebrate model. Antonie Van Leeuwenhoek. 2016;109(9):1253-9.

22. Yang J, et al. Prevalence of diverse clones of vancomycin-resistant Enterococcus faecium ST78 in a Chinese Hospital. Microb Drug Resist. 2016;22(4):294-300.

23. Drolia R, et al. Listeria adhesion protein induces intestinal epithelial barrier dysfunction for bacterial translocation. Cell Host Microbe. 2018;23(4):470-84.

24. Larsen $\mathrm{N}$, Jespersen L. Expression of virulence-related genes in listeria monocytogenes grown on danish hard cheese as affected by $\mathrm{NaCl}$ content. Foodborne Pathog Dis. 2015;12(6):536-44.
Ready to submit your research? Choose BMC and benefit from:

- fast, convenient online submission

- thorough peer review by experienced researchers in your field

- rapid publication on acceptance

- support for research data, including large and complex data types

- gold Open Access which fosters wider collaboration and increased citations

- maximum visibility for your research: over 100M website views per year

At BMC, research is always in progress.

Learn more biomedcentral.com/submissions 